\title{
Utilizing Smart Textiles-Enabled Sensorized Toy and Playful Interactions for Assessment of Psychomotor Development on Children
}

\author{
Mario Vega-Barbas, ${ }^{1,2}$ Iván Pau, ${ }^{2}$ Javier Ferreira, ${ }^{1,2,3}$ Evelyn Lebis, ${ }^{4}$ and Fernando Seoane ${ }^{1,3}$ \\ ${ }^{1}$ School of Technology and Health, KTH Royal Institute of Technology, 14152 Huddinge, Sweden \\ ${ }^{2}$ School of Telecommunications Systems and Engineering, Universidad Politécnica de Madrid, Carretera Valencia Km 7 , \\ 28038 Madrid, Spain \\ ${ }^{3}$ Faculty of Care Science, Work Life and Social Welfare, University of Borås, 50190 Borås, Sweden \\ ${ }^{4}$ Saturday Light Fever AB, 50437 Borås, Sweden
}

Correspondence should be addressed to Mario Vega-Barbas; mariovb@kth.se

Received 9 January 2015; Accepted 16 April 2015

Academic Editor: Alain Pauly

Copyright (c) 2015 Mario Vega-Barbas et al. This is an open access article distributed under the Creative Commons Attribution License, which permits unrestricted use, distribution, and reproduction in any medium, provided the original work is properly cited.

\begin{abstract}
Emerging pervasive technologies like smart textiles make it possible to develop new and more accessible healthcare services for patients independently of their location or time. However, none of these new e-health solutions guarantee a complete user acceptance, especially in cases requiring extensive interaction between the user and the solution. So far, researchers have focused their efforts on new interactions techniques to improve the perception of privacy and confidence of the people using e-health services. In this way, the use of smart everyday objects arises as an interesting approach to facilitate the required interaction and increase user acceptance. Such Smart Daily Objects together with smart textiles provide researchers with a novel way to introduce sophisticated sensor technology in the daily life of people. This work presents a sensorized smart toy for assessment of psychomotor development in early childhood. The aim of this work is to design, develop, and evaluate the usability and playfulness of a smart textile-enabled sensorized toy that facilitates the user engagement in a personalized monitoring healthcare activity. To achieve this objective the monitoring is based on a smart textile sensorized toy as catalyzer of acceptance and multimodal sensing sources to monitor psychomotor development activities during playtime.
\end{abstract}

\section{Introduction}

Healthcare, as we know it, is changing; the continuous trend of increasing cost associated with the increase in elderly population and the success in managing chronic diseases demands a paradigm change towards personalized and pervasive healthcare. Although advances in all technological fronts, for example, material, sensors, electronics, information, and communications, indeed allow the development of almost any e-health solution for pervasive monitoring, the complexity of such healthcare monitoring scenarios increases, making its exploitation more difficult, especially when a specific patient/user interaction is required.
The interactions represent reciprocal relationships established between users and different technological developments. That is, the interactions represent the convergence of interactive and physical contexts [1]. Thus, the main objective of the proper interaction design is to maximize the use of technological development and the satisfaction that the users obtained during this activity, reducing the negative aspects of the experience (frustration, discomfort) at the same time improving the positive (enjoyment, happiness) [2]. In the case of complex developments like e-health services, a design of a concrete interaction can influence the final acceptance of service by users. 
The study of technology acceptance determines the probability of success of a technological development prior to use by the target users [3]. This issue is particularly interesting when developing ICT services under study are directly affecting sensitive aspects of users [4]. Moreover, in the case of fragile and vulnerable users such as children, this acceptance involves the study of a complete ecosystem of users, which includes parents and caregivers. Therefore, this acceptance extends from a technologyuser relationship to a supervised relationship where there are several types of user with a broader set of requirements.

Previous work on how to promote acceptance in the context of sensitive service determined that by promoting a state of confidence it is possible to increase the final acceptance. Trust is ensured through the use of intuitive elements, easy to understand, and guaranteeing an optimal degree of control by users [5]. The use of smart textiles presents an interesting option for the development of sensitive services. The ability to integrate smart textiles with everyday objects (clothes, toys, etc.) leverages the familiarity and intuitiveness of the objects in which they are integrated enabling them as friendly interfaces for the deployment of ICT services.

When targeting the assessment of child development in the specific scenario of children smart textiles-enabled sensors empowering augmented toys seems to be the precise combination to monitor the key parameters required to assess child development while the child is in playful daily and accepted activity. In this study, a sensorized solution encapsulated in a toy using smart textile technology has been designed and deployed. The sensorized smart toy intends to provide a playable interaction and user experience to ensure optimal acceptance by all users involved in a daily activity like playing. Thus, the aim of this work is to design, develop, and evaluate the usability and playfulness of a smart textile-enabled sensorized toy that facilitates the children engagement in a personalized monitoring healthcare activity. Since the toys will be customized to specific applications in later stages, the toys need to have a general processing platform to change their behavior according to the final application requirements. The technical details of the processing platform including the textile sensors, digital processing, and light and sound actuators are also presented. The paper also presents a technical validation demonstrating the capabilities and feasibility of this processing part. The technical validation demonstrating the capabilities and feasibility of this processing part is another aim of this paper.

The rest of this work is organized as follows. Section 2 presents an overview of previous research efforts oriented to design and develop sensors based on toys and architectures to support sensitive services. Then, Section 3 describes the design and development of the smart toy and the communication between it and the smart space. The evaluation of playfulness and acceptance by users is analyzed in Section 4 and, finally, Sections 5 and 6 summarize the discussion and conclusions of this work.

\section{Background}

Child development in the early years is characterized by the progressive acquisition of important functions such as postural control, autonomy of movement, communication, body language, and social interaction [6]. Early detection of warning signs, which are potential indicators of problems in child development, is a key issue because it offers greater guarantees to prevent added pathologies, achieving functional improvements, and enable a more adaptive fit between a child and his environment $[7,8]$. Thus, telemedicine not only offers the possibility of providing health services to children who live in isolated areas [9], but also can provide novel monitoring and diagnostic methods that may be beneficial for the children's health.

An interesting trend related with the scope of this research work in telemedicine and telepediatrics is providing pervasive services and ubiquitous solutions that enable experts to observe and treat children in their natural environment, preferably homes or childhood centers $[10,11]$. However, the potential of telemedicine in concrete telemonitoring in smart spaces has not yet been reflected in a significant implementation [12-14]. Besides challenges not only are related to current status of the technology but also are associated with other factors related to the inclusion of technology in areas such as organization and coordination of providers, updating healthcare processes, or acceptance of health services by users $[15,16]$. This last factor, the acceptance, is especially critical due to the characteristics of health services: privacy, safety, reliability, and intention of use.

Also, an important amount of researchers involved in technologies for physiological measurements and personalized healthcare monitoring focuses their efforts on developing sensors based on smart textile materials [17-20]. Smart textiles offer benefits related to the intuitiveness ensured by the use of known interaction and accepted elements by all human beings. For example, in [21-23] the authors develop and use different set of clothes for recording measurements for different applications of assessment of fluid distribution in the body. In [24] researchers attempt to include monitoring of daily activities of people using smart textiles; specifically this research shows how to assess the stress in real time on workers under extreme conditions. Finally, [25] shows an overview about the usefulness of developing textile interfaces as a means of interaction. However, when the target set of users are children, the use of clothing presents problems related to the unpredictable behavior of these.

In this sense, the creation of playful interactions presents a potential and interesting method to get children involved in some telemedicine and healthcare monitoring activities [2628]. Currently, part of the research in this field is aimed at developing serious games that use smartphones and tablets as a tool (Bring Your Own Device or BYOD) [29]. In case of children, especially aged between 0 and 6 years, toys represent the most intuitive BYOD. The development of smart toys seems to be a useful tool for monitoring their daily activity because they are safe and enjoyable for very small children [30]. In this way, a smart toy represents a target element which connects children with the telemonitoring 
service and in which we can deploy playful interactions to improve the engagement of such specific type of user.

As we present below, the literature related to the development of intelligent toys shows two clear trends. First is toys and smart spaces designed for the treatment of chronic diseases such as autism. Furthermore, the research focuses on the educational field, developing environments, and interaction elements based on toys to improve learning processes.

Some examples of efforts related with the first trend are found [30-32]. In [31] the researchers present a complete development of a toy system based on smart textiles for general use. Such work represents a first approach to the use of smart textiles for the development of interactions that promote free play, that is, that simulate traditional toys, encourage creativity in children, offer interactivity, and encourage social interaction. The work in [30] shows a comprehensive solution for identifying and monitoring recording activities related to the game in order to identify possible developmental disorders of children. In that work, authors define three elements, a set of intelligent toys that make up a wireless sensor network, a recognition patterns system of behavior, and a query interface for further analysis of the data obtained. The last two elements of the system employ direct recording of the activities of the children. Complementing this work, [32] offers the possibility of inclusion of a robot in child domain. This robot provides facilities for communication and social interaction for children with certain types of disabilities. This robot has the ability to organize games that encourage social skills of children associated with social relationships.

Finally, relevant research works related with the second trend are analyzed in [33-35]. These articles studied how toys computationally enhanced can provide improvements in learning and psychomotor development of children. The system proposed in [33] is designed to create a sixth sense of health through pervasive components located around to detect information about the welfare of children. The novelty of this experiment is to provide a pervasive ecosystem component plug-and-play that is geared towards health education and children's autonomy through an approach of "Do-itYourself." Authors in [34] show how the interaction with toys can help to promote and support the psychomotor skills of children during their development. Using kinesthetic methods, that is, toys and materials that use the movement to support communication and learning through computational physics interaction, it is possible to establish healthy and profitable relationships between children and technology with an educational or therapeutic target. Finally, in [35], soft toy design is presented, using conductive fabrics and pressure detection circuits. The system is a highly sensitive deformable controller, which can be flexibly used in a range of settings for the continuous or discrete control. The controller is wireless and can be used to expand the interactive possibilities of mobile computing devices. Multiple controllers can also be networked in collaborative scenarios.

\section{Sensor-Toy Design}

The development of toys for children aged between 0 and 6 must provide interaction of free play. As the authors of [31] exposed, this interaction should favor four factors:

(i) it should simulate a traditional toy,

(ii) it should encourage creativity in children during use,

(iii) it has to be interactive,

(iv) it encourages social interaction.

In this sense, smart textiles gain importance because they allow design and build toys with a traditional shape and texture. Furthermore, the design of a smart toy should have a clear functional objective, which in this case is to record a set of physiological measurements to allow the assessment of children's psychomotor development through the use of the toy. Again, this goal is carried out with the use of smart textiles utilizing their potential to create textile sensors, which can be integrated, in the smart toy in a seamless way.

3.1. Set of Physiological Measurements. The set of physiological measurements that can be evaluated for a correct assessment of psychomotor development of a child from birth to an age limit of 6 is varied [36]. As aforementioned, one of the objectives of the presented work is to provide a sensor that can be encapsulated in a toy. In addition, the smart textile sensor intends to provide a playable interaction and user experience to ensure optimal acceptance by all users involved in a daily activity like playing.

The behavior of a child during a playing activity with traditional toys shows that an objective measure is the force that children develop in their hands [37]. This force can be decomposed into force of compression and stretching. It is also interesting for children developmental experts to know if children react to reactive stimuli such as sounds or lights $[38,39]$. These stimuli are easily relatable to the abovementioned movements, establishing a feedback interaction between the detection of force on the toy and the production of sound and light effects.

3.2. Sensing Toy Shape and Materials. The first result of this research work is a sensorized prototype toy based on interaction design foundations and build using smart textile sensors and the lilypad arduino platform. The toy was designed to measure two psychomotor abilities, handgrip and stretch force.

The previous analysis of the background and related literature gathered the initial requirements to develop this smart toy.

(i) Cloth texture is preferable than a plastic or wire texture.

(ii) The interaction must report a feedback to the children in order to attract them to use the toy repeatedly, using lights, sounds, and vibrations.

(iii) The battery must be hidden and well protected. 


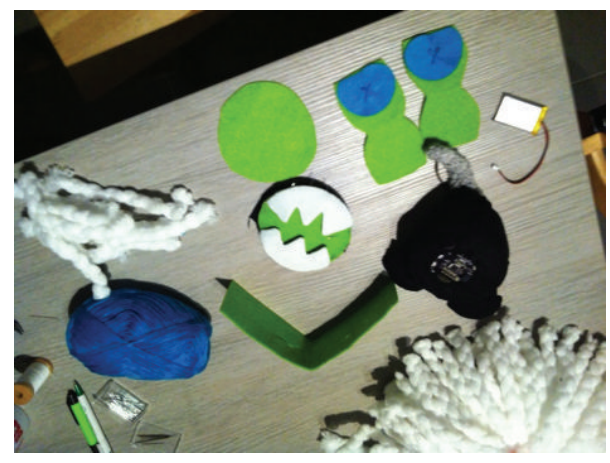

FIGURE 1: Example of the smart toy pieces, both textile elements, electronics, and battery.

(iv) The shape and colors should be neutral in relation to the children gender.

(v) The toy must send the information gathered to a sink in order to be analyzed by experts.

Two types of textile-based sensors were used to gather the hand-palm pressure and body strength using a textile pressure sensor and a textile stretch sensor. This way, these two sensors make it possible to assess the handgrip and stretch activity correspondingly. These sensors were placed in a smart toy composed of two elements: a ball which includes the first textile sensor and a monster toy which includes the second one.

The first pressure sensor was made with Velostat film of $2 \times 1$, stripes of conductive Medtex $130 \mathrm{Ag}$ Nylon stretch fabric manufactured by Statex, and Scotch tape. In addition, this sensor was connected to an RGB-LED, a buzzer, and a vibrating actuator to provide sound and visual feedback to the users. When the pressure sensor is pressed by a hand gesture, a change in resistance is converted into a voltage change that is sensed on the lilypad, processed, and communicated.

The second smart textile sensor developed was the stretch sensor. This sensor was made with a hand knitted fabric made of semiconductive yarn that changes its electrical properties when it is stretched and released. A piece of $3 \times 7 \mathrm{~cm}$ cloth knitted with conductive $\mathrm{Nm} \mathrm{1/3}$ wool yarn ending in both sides with conductive Medtex $130 \mathrm{Ag}$ nylon stretchable fabric material was used as sensing area. Figures 1 and 2 show an example of this process. Both textile-based sensors were connected to the analog inputs in the lilypad using a simple voltage divider [40], where the pull-down resistor value was calculated to obtain the maximum sweep range from the textile sensors.

The textile sensors and conventional fabrics were sewed into a toy, similar to other common toys, and the final result is shown in Figure 3. An example of the measurements obtained with the toy is shown in Figure 4. These measurements show how the sensorized smart toy digitizes and processes the data gathered from smart textiles sensors.

3.3. Data Communication. The use of the toy by children must be recorded and stored in a database for further processing. To enable wireless data communication between

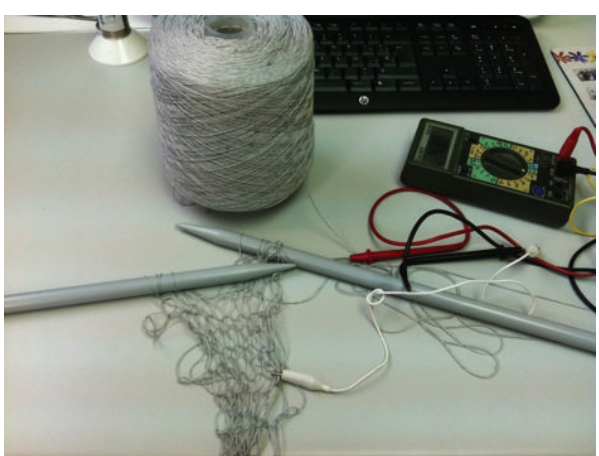

FIgURE 2: An example of the developing of a stretch sensor using smart textiles.

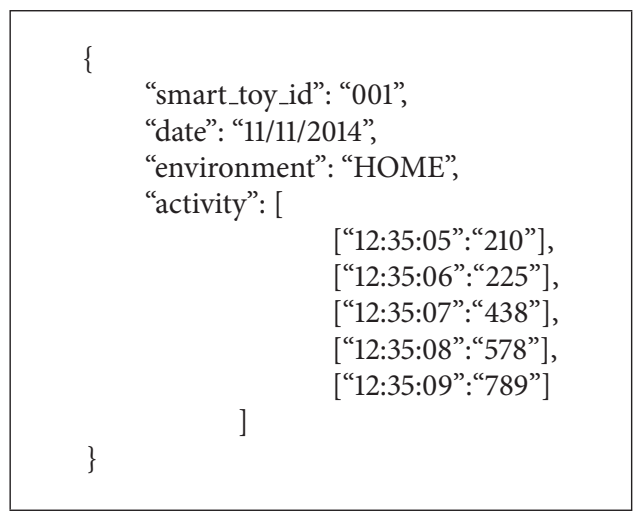

Box 1: Representation of information processed by the smart toy for storing in the database.

the toy and the external host a lilypad XBee wireless module has been used. The data containing the activity of the smart toy is encapsulated in string messages. Whenever a strength or stretch even is detected a new message is sent to the sink with the following format:

\section{ST_ID\#stgth:ANALOG_OUTPUT\#sttch: ANALOG_OUTPUT}

and a sample of it:

001\#stgth:567\#sttch:18

Here ST_ID refers to the smart toy used. If we develop a system with several smart toys this tag contradistinguishes between them. The stgth tag represents the strength and provides the analog output catch by the lilypad board. In the same way, the sttch tag provides the analog output for the stretch make on the smart toy.

Finally, this information is stored in a database oriented to documents. This kind of databases provides a global view of all activities and actions generated during the interaction with the toy. The sink element gathers context information such as the date, time, environment, and info and adds to the data from the smart toy. Thus, the information stored is presented to physicians and caregivers in an understandable way. A sample of this information is detailed hereafter (see Box 1). 


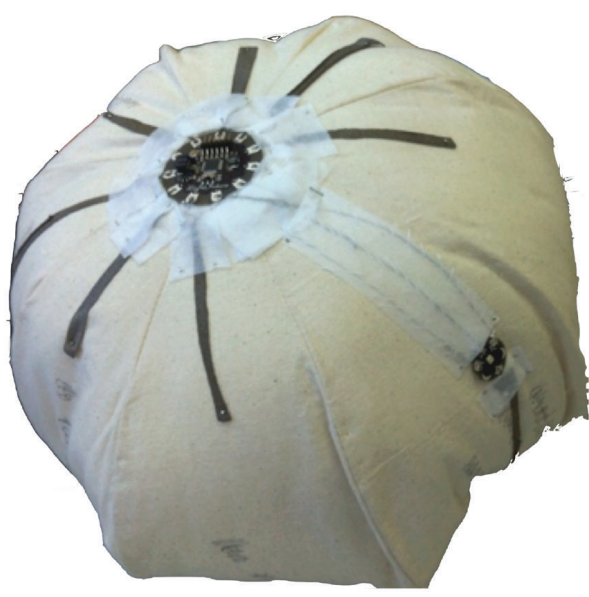

(a)

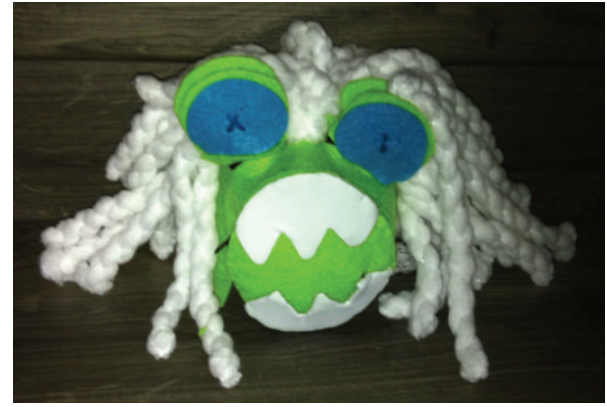

(b)

Figure 3: Smart toy developed in its final version. (a) The ball toy. (b) The monster toy.

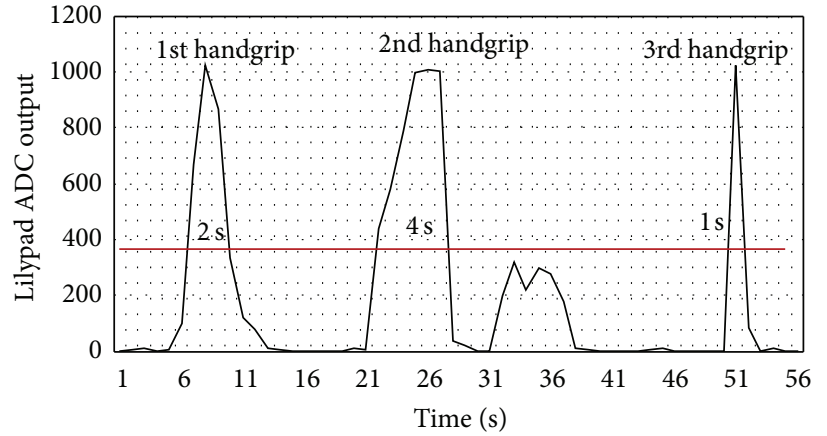

(a)

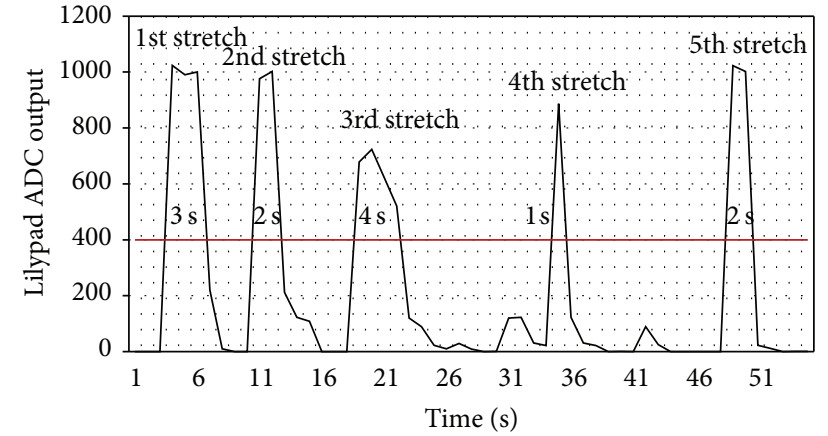

(b)

FIGURE 4: Graphical representation of data gathered by smart textile sensors included in the smart toy: (a) handgrip measure output data gathered from the press sensor and (b) stretch measure output data gathered from stretch sensor. In both cases the data is produced by the output of the arduino internal ADC available in the lilypad bounded between 0 and 1023. The red line represents the threshold that determines the existence of activity or not. The value of the threshold was defined by direct observation during the child-toy interaction.

As we can observe in Figure 5, the communication process is organized as follows. The smart toy transmits the gathered information to a receiver formed by an arduino UNO with a wireless Zigbee communication module and an Ethernet module. Once generated, an information packet is sent to the database in the server at school. The information stored in the database at school is replicated via Internet to a database at the supervision center allowing experts to have access to all data gathered if they need.

\section{Evaluation of Playfulness}

To achieve an evaluation of the sensorized smart toy, a field observation based on questionnaires to measure the user experience was designed. The aim of this observation was to validate the use of the toy from a final user point of view in order to know if the produced sensorized smart toy meets the design requirements, in other words, if the sensorized smart toy can record a set of physiological measurements while providing a playfulness experience for the children.

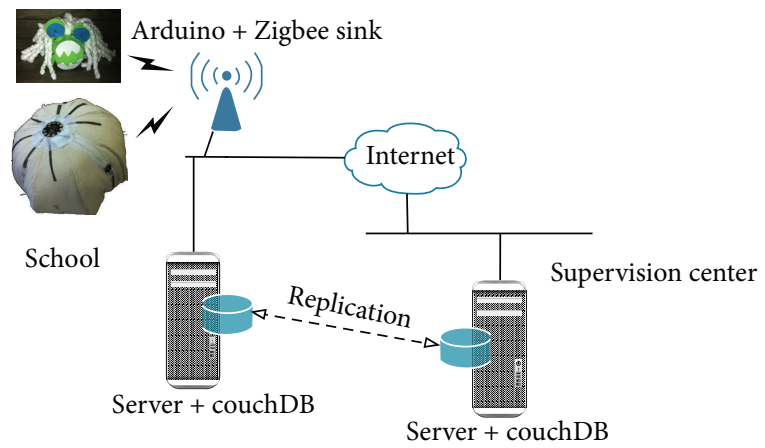

FIGURE 5: Overall deployment diagram that specifies the communication process.

With such purpose in mind, a user experience experiment (UX-experiment) was designed with the specific purpose to observe if the smart toy fits our previous expectations according to children use, understanding, acceptance, and, 
TABLE 1: Structure of the questionnaire.

\begin{tabular}{|c|c|c|}
\hline Evaluation area & Target aspect of the smart toy & Question \\
\hline \multirow{4}{*}{ Playfulness } & Acceptance & Children want to use the toy \\
\hline & Understanding & Children understood the smart object as a toy \\
\hline & Intuitiveness & The smart toy seemed intuitive \\
\hline & Environment cohesion & There were many differences of shape with other toys in the playground \\
\hline \multirow{2}{*}{ Caregiver confidence } & User comfort & Children used the smart textile sensors in the smart toy \\
\hline & Caregiver confidence & Teachers felt confident with the use of the toy by children \\
\hline \multirow{2}{*}{ Social interactions } & Innovation capabilities & Children discovered new ways to play with the toy \\
\hline & Smart toy integration & The toy was integrated successfully in the playground \\
\hline
\end{tabular}

therefore, playfulness. This experiment started with the definition of a questionnaire to conduct the field observation. Next we carried out a direct observation in a nursery school involving the teachers in this process.

4.1. Questionnaire Definition. The questionnaire used in this experiment was based on previous work such as the IBM Computer Usability Satisfaction Questionnaire (CUSQ) and rules proposed by the System Usability Scale (SUS). To obtain useful information about how the toy seems interesting for children, the questions were adapted accordingly in these methods. The number of questions was reduced to 8 in order to ease the experiment and avoid a boring experience for both teachers and children.

The questions defined were organized in 3 evaluation areas, which included the functional objectives of the research work. Playfulness defines if any children could use the smart toy as a toy. The caregiver confidence refers to requirements from a medical point of view; that is, the smart toy can be used for physiological monitoring. Finally, the social interaction area includes questions about how the smart toy provides ways of interaction among children in a gaming environment and encourages creativity in the children. Table 1 summarizes this organization.

4.2. User Experience Test. The questionnaire was used to conduct the user experience test (UX). The test was filled out with the collaboration of 10 children $(N=10)$ at the age between 3 and 6 years (mean $=4.5, \mathrm{SD}=0.69)$, always under supervision of their teachers. The experiment was carried out in a real playground environment.

Each child played with the toy during 20 minutes under supervision of the teachers and a researcher. Along this time the teachers filled out the questionnaire marking the previous questions with a grade between 1 (strongly disagree) and 5 (strongly agree). Finally, the test was anonymous and balanced in gender ( 5 girls and 5 boys).

4.3. Evaluation of UX Experiment. The UX experiment gathered data from the observation of 10 children, 5 boys and 5 girls, playing with the smart toy prototype in a playground at the school. Also the teachers contributed to the experiment supervising the entire process and providing comments to each question. Next Figure 6 shows the general results of this

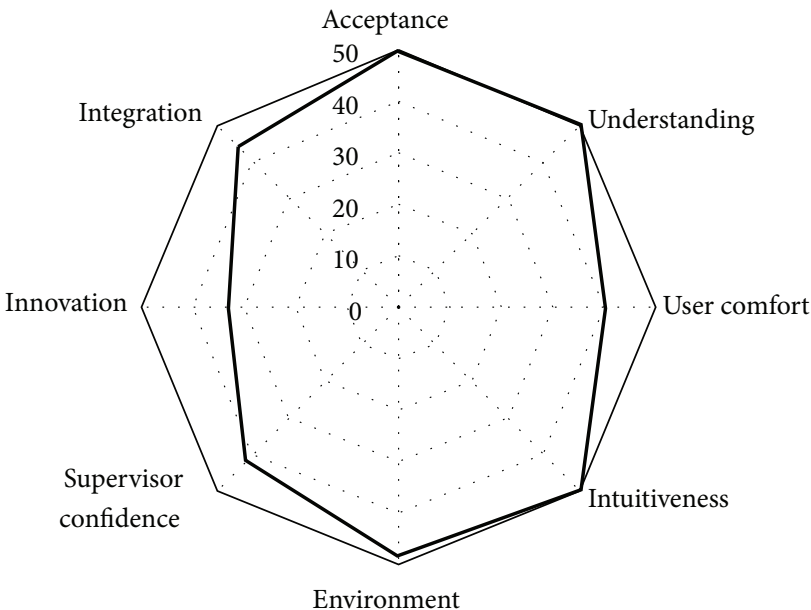

FIGURE 6: Graphical representation of the global results of the UX experiment.

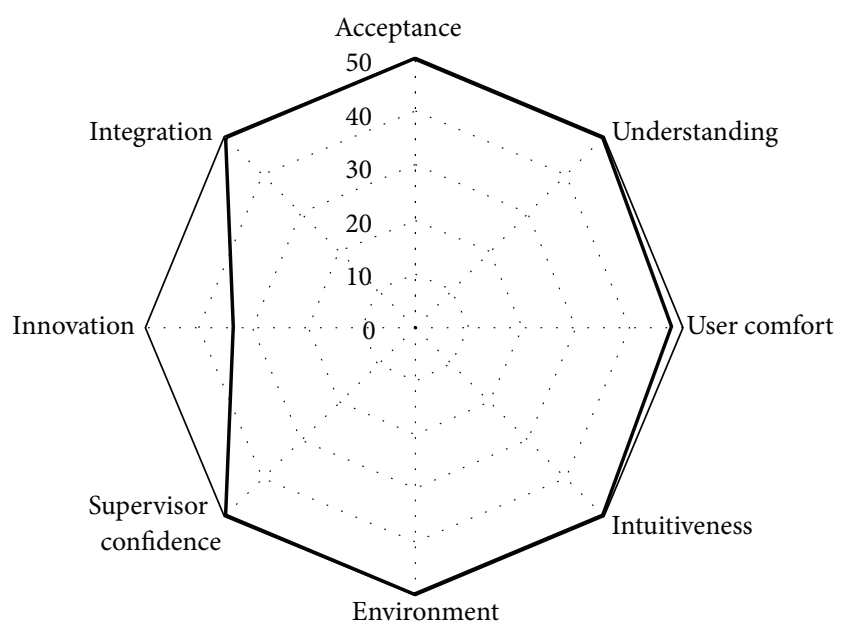

FIGURE 7: Graphical representation of the girls' results of the UX experiment.

experiment and the results per gender are shown in Figures 7 and 8.

The results show that $100 \%$ of children understood the smart object as a toy (understanding) without any extra explication (intuitiveness) and wanted to use it to play 


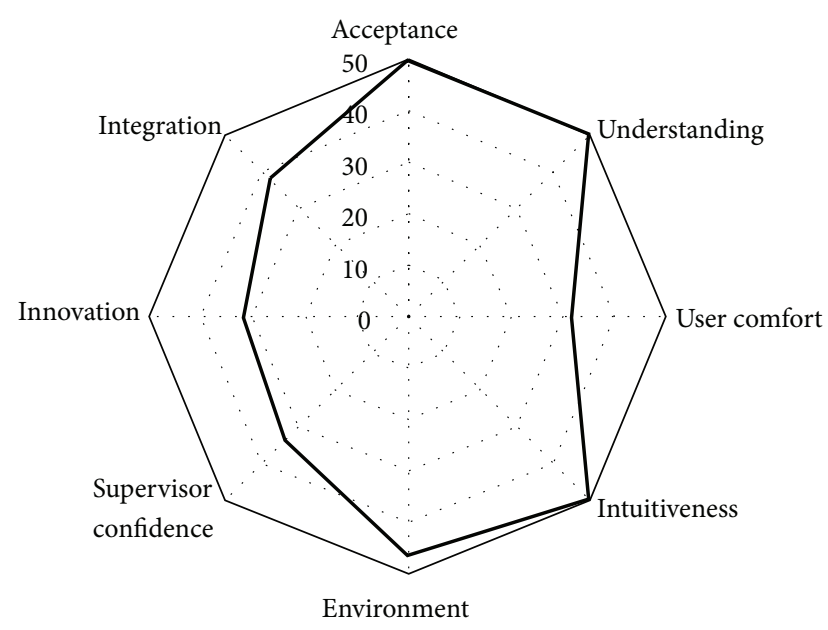

FIGURE 8: Graphical representation of the boys' results of the UX experiment.

(acceptance). However, boys encountered problems to use the toy in the way that it was designed (user comfort) since only in $64 \%$ of the chances the textile sensors were used while girls discovered the function of the textile sensor more often, with a ratio of usage of $96 \%$. Also, boys found some problem in integrating the toy with their other toys (environment) but not the girls. Finally the ratio of discovering new ways to play with the toy (innovation) was $66 \%$, similar in boys and girls. The teachers explained that the children wanted to play with the sensorized smart toy only but some child invented new games such as creating new melodies. In this way, the teachers felt a grade of confidence of $84 \%$ with the use of the toy, with a strong difference between boys (68\%) and girls (100\%).

In addition, it was observed that the children played individually and in group with the toy but not for a long period of time (20-25 minutes). The girls used the toy exactly the way it was designed, using the smart textiles sensors, whereas the boys accidently discovered the smart functions. Amongst all children the hair of the toy and the lights were the most appealing feature to them.

Finally, from the discussion with teachers, information about the confidence of the informal caregivers was obtained. The teachers noted that the toy was a useful tool to integrate children with special need. For example, they tried to include a shy child into the group through the smart toy and succeeded. However, the teachers felt uncomfortable letting the toy out of their sight because some children, boys, played too rough and therefore they were worried about the possibility that children could break the sensorized smart toy. The observation ended when the children started watching a movie. The toy was placed at the end of the matrass with its eyes facing towards the screen.

\section{Discussion}

The reported work shows that is possible to develop a sensorized smart toy based on emerging technologies as smart textile sensors facilitating personalized healthcare

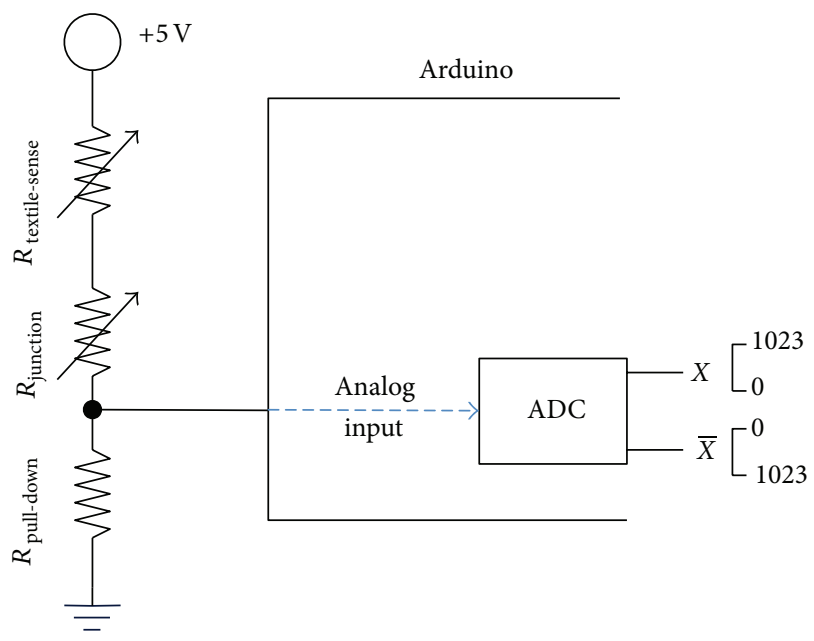

FIGURE 9: Electrical circuit equivalent of the sensors implemented in the smart toy. Notice the presence of $R_{\text {junction }}$ and its contribution to the voltage sense at the analog input of the Arduino. The dynamic range of the $\mathrm{ADC}$ can be adjusted to voltage change expected on the sensor by software. In addition the ADC digital output $X$ and its complement $\bar{X}$ can be selected also.

monitoring and increasing the acceptance of the solution by transforming such activity in a playful interaction. In particular this research presents a smart toy, which can monitor activities related with specific aspects related to childhood development using smart textiles and electronics as well as information and communications technologies.

Both textile sensors developed use a very straightforward electronic implementation with a simple voltage divider and a pull-down resistor. The direct connection of the analog input of the Arduino to the textile sensor implies that the sensing input of the Arduino will sense not only changes in the resistance of the textile element but also changes in the impedance of the junction; therefore none of the sensors will be able to produce precise readings. See Figure 9.

The intended purpose of these specific smart sensing toys was not to obtain a precise representation of a physical magnitude per se, for example, pressure, elongation, or force, but the detection of a specific activity directly related to the change in the sensed physical magnitude. Therefore since such simplistic sensor implementation provides handgrip and stretching data useful for assessing the existence of activity and the length of the given activity, we concluded that actual implementation of the sensors is adequate for the targeted application.

Any other electrical implementation would increment the number of passive and active component increasing the cost and the complexity unnecessarily, but, in case that the monitoring application required better sensing performance, there are other textile-enabled solutions for sensing pressure [41] and stretch [42] currently available.

In short, developing enhanced toys using smart textiles sensors makes it possible to establish a link between the virtual world represented by pervasive technology and the physical world where children live, a key factor in Early 
Childhood Intervention [7]. That means we can take advantage of benefits of a complex and abstract technology such as ubiquitous computing without renouncing to usability, acceptation, and confidence offered by everyday objects such as toys [43]. This way, the use of textiles to develop this type of smart objects fosters technology acceptance enabling novel e-health applications.

Finally, we consider that there are several applications that could benefit from the use of sensorized smart toys ranging from rehabilitation activities to support treatment of children with autism [44] in a similar manner to what robotic toys do [45].

\section{Conclusions}

New computing paradigms such as ubiquitous computing and emerging technologies as smart textiles offer potential ways of interaction that favor the creation of new types of services that directly affect complex tasks such as ehealth processes, making them simpler and more accessible. However, this type of technology, useful and necessary, is often rejected due to implications of confidence, privacy, and security. More often this rejection happens when users involved are children and the services offered use sensible and private data such as medical monitoring.

Thus, smart toys provide an interesting way to generate the necessary confidence to ensure the use and acceptance of these solutions. Toys represent an intuitive mode of communication and interaction with children in young ages. A suitable use of these tools could make easy other processes that are presumptively complex and critical. In this sense, the smart toy shows us how to orchestrate these types of tools to ensure the children acceptance and, therefore, the use of the implemented services.

This research line aims to evaluate the usefulness of smart toys to improve the user acceptance of critical ehealth services. Aligned with that aim this work proves that it is possible to develop and deploy a sensorized smart toy that supports and increases the confidence of the final users involved in the monitoring for assessment of psychomotor development during early childhood.

\section{Conflict of Interests}

Mario Vega-Barbas, Iván Pau, and Evelyn Lebis declare no conflict of interests regarding the publication of this paper. Fernando Seoane and Javier Ferreira are both founders and partly owners of Z-Health Technologies AB.

\section{Acknowledgments}

The work for this study has been funded by the Spanish Ministry of Economy and Competitivity under Grant no. BES-2011-044568. In addition, this research work has been developed within the project EDUCERE under Grant no. TIN2013-47803 by Spanish Ministry of Economy and Competitivity.

\section{References}

[1] J. Lowgren, "Interaction design-brief intro," in The Encyclopedia of Human-Computer Interaction, M. Soegaard and R. F. Dam, Eds., The Interaction Design Foundation, Aarhus, Denmark, 2nd edition, 2014, https://www.interactiondesign.org/encyclopedia/interaction_design.html.

[2] H. Sharp, Y. Rogers, and J. Preece, Interaction Design: Beyond Human-Computer Interaction, 2002.

[3] F.-Y. Pai and K.-I. Huang, "Applying the Technology Acceptance Model to the introduction of healthcare information systems," Technological Forecasting and Social Change, vol. 78, no. 4, pp. 650-660, 2011.

[4] K. Connelly, "On developing a technology acceptance model for pervasive computing," in Proceedings of the 9th International Conference on Ubiquitous Computing (UBICOMP)_Workshop of Ubiquitous System Evaluation (USE '07), p. 520, Springer, Innsbruck, Austria, September 2007.

[5] M. Vega-Barbas, I. Pau, and F. Seoane, "Confidence: dependencies and their critical role in fostering user acceptance in pervasive applications," in Proceedings of the EAI 4th International Conference on Wireless Mobile Communication and Healthcare (Mobihealth '14), pp. 283-286, IEEE, Athens, Greece, November 2014.

[6] J. P. Shonkoff and S. J. Meisels, Handbook of Early Childhood Intervention, Cambridge University Press, Cambridge, UK, 2000.

[7] V. Soriano and European Agency for Development in Special Needs Education, "Early childhood intervention: analysis of situations in europe; key aspects and recommendations," Summary Report, European Agency for Development in Special Needs Education, 2005.

[8] Y. Zheng, S. P. Maude, and M. J. Brotherson, "Early childhood intervention in China," Journal of International Special Needs Education, vol. 18, no. 1, pp. 29-39, 2015.

[9] W. B. Karp, R. K. Grigsby, M. McSwiggan-Hardin et al., "Use of telemedicine for children with special health care needs," Pediatrics, vol. 105, no. 4 I, pp. 843-847, 2000.

[10] J. P. Marcin, "Telemedicine in the pediatric intensive care unit," Pediatric Clinics of North America, vol. 60, no. 3, pp. 582-592, 2013.

[11] M. L. M. Ruiz, V. Á. V. Duboy, C. T. Loriente, and I. P. de la Cruz, "Evaluating a web-based clinical decision support system for language disorders screening in a nursery school," Journal of Medical Internet Research, vol. 16, no. 5, article e139, 2014.

[12] C. Krüger and M. Niemi, "A telemedicine network to support paediatric care in small hospitals in rural Tanzania," Journal of Telemedicine and Telecare, vol. 18, no. 1, pp. 59-62, 2012.

[13] L. Kidd, S. Cayless, B. Johnston, and Y. Wengstrom, “Telehealth in palliative care in the UK: a review of the evidence," Journal of Telemedicine and Telecare, vol. 16, no. 7, pp. 394-402, 2010.

[14] T. H. F. Broens, R. M. H. A. Huis in't Veld, M. M. R. Vollenbroek-Hutten, H. J. Hermens, A. T. van Halteren, and L. J. M. Nieuwenhuis, "Determinants of successful telemedicine implementations: a literature study," Journal of Telemedicine and Telecare, vol. 13, no. 6, pp. 303-309, 2007.

[15] C. Sanders, A. Rogers, R. Bowen et al., "Exploring barriers to participation and adoption of telehealth and telecare within the Whole System Demonstrator trial: a qualitative study," BMC Health Services Research, vol. 12, no. 1, article 220, 2012. 
[16] G. Demiris, N. Charness, E. Krupinski et al., "The role of human factors in telehealth," Telemedicine and e-Health, vol. 16, no. 4, pp. 446-453, 2010.

[17] J. Löfhede, F. Seoane, and M. Thordstein, "Textile electrodes for EEG recording — a pilot study," Sensors, vol. 12, no. 12, pp. 1690716919, 2012.

[18] J. C. Márquez, F. Seoane, E. Välimäki, and K. Lindecrantz, "Comparison of dry-textile electrodes for electrical bioimpedance spectroscopy measurements," Journal of Physics: Conference Series, vol. 224, no. 1, Article ID 012140, 2010.

[19] J. C. Marquez, J. Ferreira, F. Seoane, R. Buendia, and K. Lindecrantz, "Textile electrode straps for wrist-to-ankle bioimpedance measurements for Body Composition Analysis. Initial validation \& experimental results," in Proceedings of the 32nd Annual International Conference of the IEEE Engineering in Medicine and Biology Society (EMBC '10), pp. 6385-6388, IEEE, September 2010.

[20] L. Xie, G. Yang, L. Xu, F. Seoane, Q. Chen, and L. Zheng, "Characterization of dry biopotential electrodes," in Proceedings of the 35th Annual International Conference of the IEEE Engineering in Medicine and Biology Society (EMBC '13), pp. 1478-1481, July 2013.

[21] J. C. Márquez, F. Seoane, and K. Lindecrantz, "Textrode functional straps for bioimpedance measurements-experimental results for body composition analysis," European Journal of Clinical Nutrition, vol. 67, no. 1, pp. S22-S27, 2013.

[22] I. Cuba-Gyllensten, P. Gastelurrutia, J. Riistama et al., "A novel wearable vest for tracking pulmonary congestion in acutely decompensated heart failure," International Journal of Cardiology, vol. 177, no. 1, pp. 199-201, 2014.

[23] J. Hännikäinen, T. Vuorela, and J. Vanhala, "Physiological measurements in smart clothing: a case study of total body water estimation with bioimpedance," Transactions of the Institute of Measurement and Control, vol. 29, no. 3-4, pp. 337-354, 2007.

[24] F. Seoane, I. Mohino-Herranz, J. Ferreira et al., "Wearable biomedical measurement systems for assessment of mental stress of combatants in real time," Sensors, vol. 14, no. 4, pp. 7120-7141, 2014.

[25] S. Dakova and N. Dumont, "An overview of textile interfaces," 2014, https://hci.rwth-aachen.de/tiki-download_wiki_attachment.php?attId=1176.

[26] E. Gabarron, T. Schopf, J. A. Serrano, L. Fernandez-Luque, and E. Dorronzoro, "Gamification strategy on prevention of STDs for youth," in Studies in Health Technology and Informatics, vol. 192, p. 1066, 2013.

[27] R. Hu, G. Fico, J. Cancela, and M. T. Arredondo, "Gamification system to support family-based behavioral interventions for childhood obesity," in Proceedings of the IEEE-EMBS International Conference on Biomedical and Health Informatics (BHI '14), pp. 181-184, IEEE, Valencia, Spain, June 2014.

[28] A. S. Miller, J. A. Cafazzo, and E. Seto, "A game plan: Gamification design principles in mHealth applications for chronic disease management," Health Informatics Journal, 2014.

[29] O. F. Roca, BYOD, Gamification \& High Definition Innovations for Telemedicine, CATAI, 2012.

[30] T. L. Westeyn, G. D. Abowd, T. E. Starner, J. M. Johnson, P. W. Presti, and K. A. Weaver, "Monitoring children's developmental progress using augmented toys and activity recognition," Personal and Ubiquitous Computing, vol. 16, no. 2, pp. 169-191, 2012.

[31] L. Berglin, "Spookies: combining smart materials and information technology in an interactive toy," in Proceedings of the
Conference on Interaction Design and Children (IDC '05), pp. 1723, ACM, Boulder, Colo, USA, June 2005.

[32] P. Marti, "Bringing playfulness to disabilities," in Proceedings of the 6th Nordic Conference on Human-Computer Interaction: Extending Boundaries (NordiCHI '10), pp. 851-856, ACM, October 2010.

[33] S. Ananthanarayan and K. A. Siek, "Health sense: a gedanken experiment on persuasive wearable technology for health awareness," in Proceedings of the 1st ACM International Health Informatics Symposium (IHI '10), pp. 400-404, ACM, November 2010.

[34] H. Raffle, "Kinesthetic media: touch, toys \& interactive materials," in Proceedings of the ACM SIGGRAPH Educators Program (SIGGRAPH '06), p. 8, ACM, Boston, Mass, USA, July 2006.

[35] M. Grierson and C. Kiefer, "NoiseBear: a wireless malleable multiparametric controller for use in assistive technology contexts," in Proceedings of the CHI '13 Extended Abstracts on Human Factors in Computing Systems (CHI EA '13), pp. 29232926, ACM, 2013.

[36] E. Matute, M. Rosselli, A. Ardila, and F. Ostrosky-Solis, Evaluación Neuropsicológica Infantil, Manual Moderno, Mexico City, Mexico, 2007.

[37] M. Semrud-Clikeman and P. Teeter, Neuropsicología Infantil. Evaluación e Intervención en los Trastornos Neuroevolutivos, UNED Pearson, Madrid, Spain, 2011.

[38] X. Wang, "Impact analysis of early environmental stimuli on infant behavior and development," Guide of China Medicine, vol. 3, article 10, 2013.

[39] W. Buckleitner, Child Development 101 for Interactive Media Designers: An Overview of Influential Theories, Applied to Practice, Active Learning Associates, Mishawaka, Ind, USA, 2015.

[40] T. Igoe, Making Things Talk: Using Sensors, Networks, and Arduino to See, Hear, and Feel Your World, O'Reilly Media, Inc., 2011.

[41] B. Zhou, J. Cheng, M. Sundholm, and P. Lukowicz, "From smart clothing to smart table cloth: design and implementation of a large scale, textile pressure matrix sensor," in Architecture of Computing Systems-ARCS 2014, pp. 159-170, Springer International, 2014.

[42] T.-W. Shyr, J.-W. Shie, C.-H. Jiang, and J.-J. Li, "A textile-based wearable sensing device designed for monitoring the flexion angle of elbow and knee movements," Sensors, vol. 14, no. 3, pp. 4050-4059, 2014.

[43] M. Kuniavsky, Smart Things: Ubiquitous Computing User Experience Design, Elsevier, New York, NY, USA, 2010.

[44] W. Farr, N. Yuill, and S. Hinske, "An augmented toy and social interaction in children with autism," International Journal of Arts and Technology, vol. 5, no. 2-4, pp. 104-125, 2012.

[45] I. Giannopulu and G. Pradel, "Multimodal interactions in free game play of children with autism and a mobile toy robot," NeuroRehabilitation, vol. 27, no. 4, pp. 305-311, 2010. 

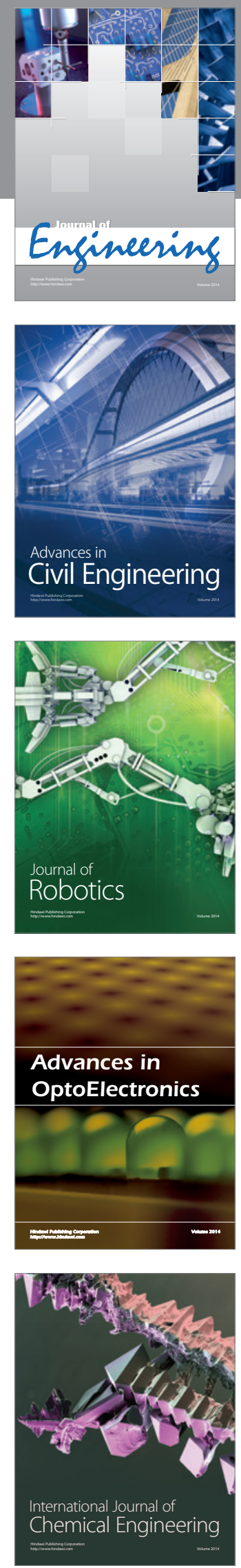

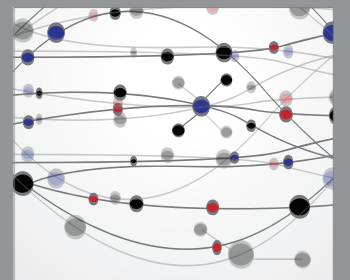

The Scientific World Journal
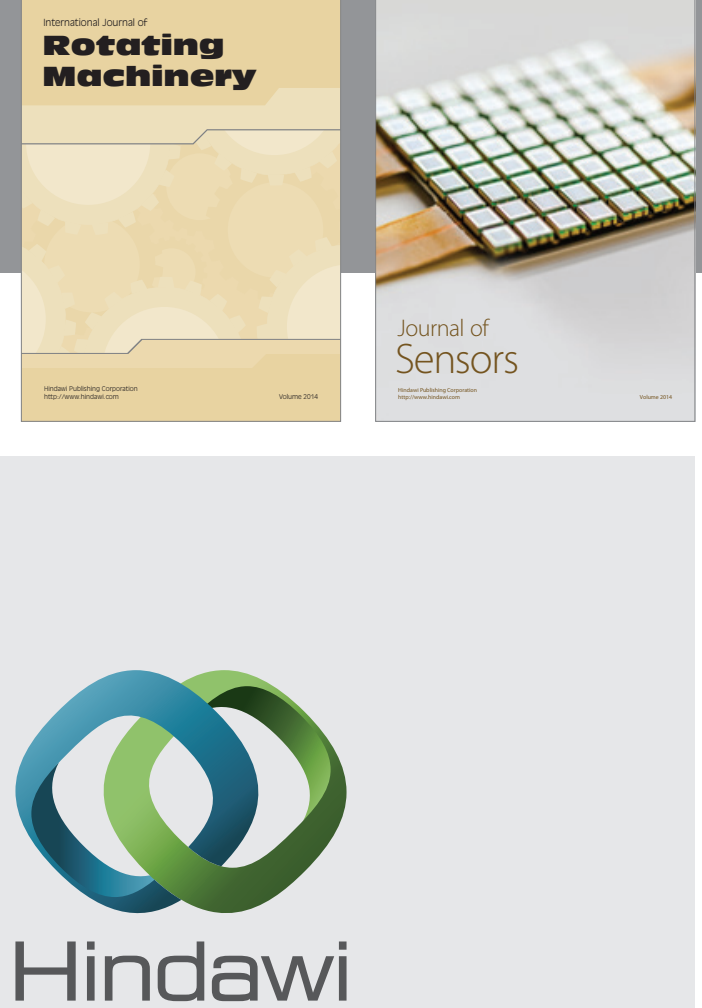

Submit your manuscripts at http://www.hindawi.com
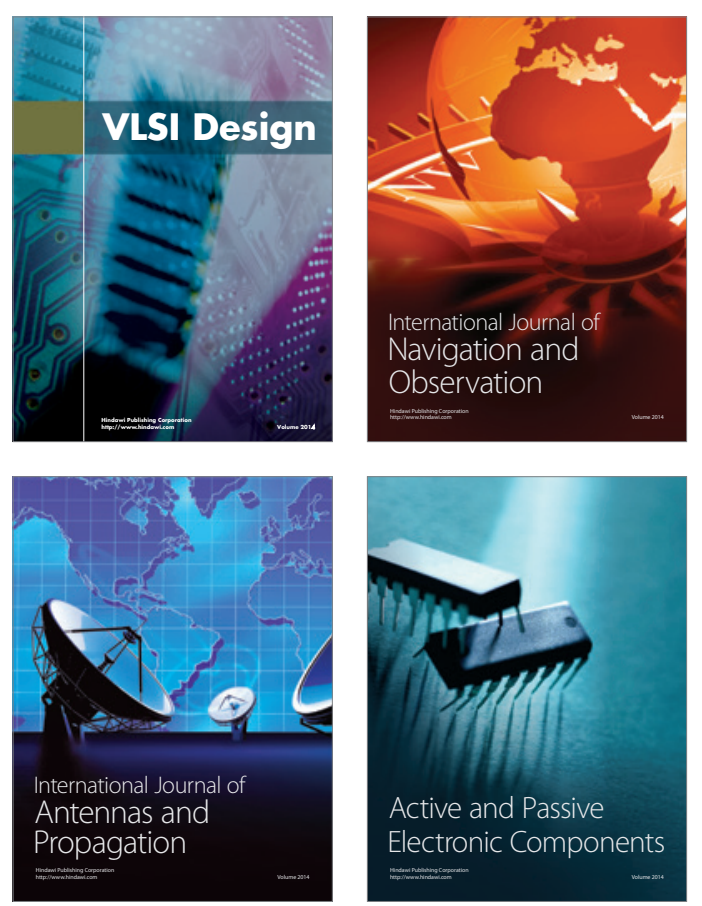
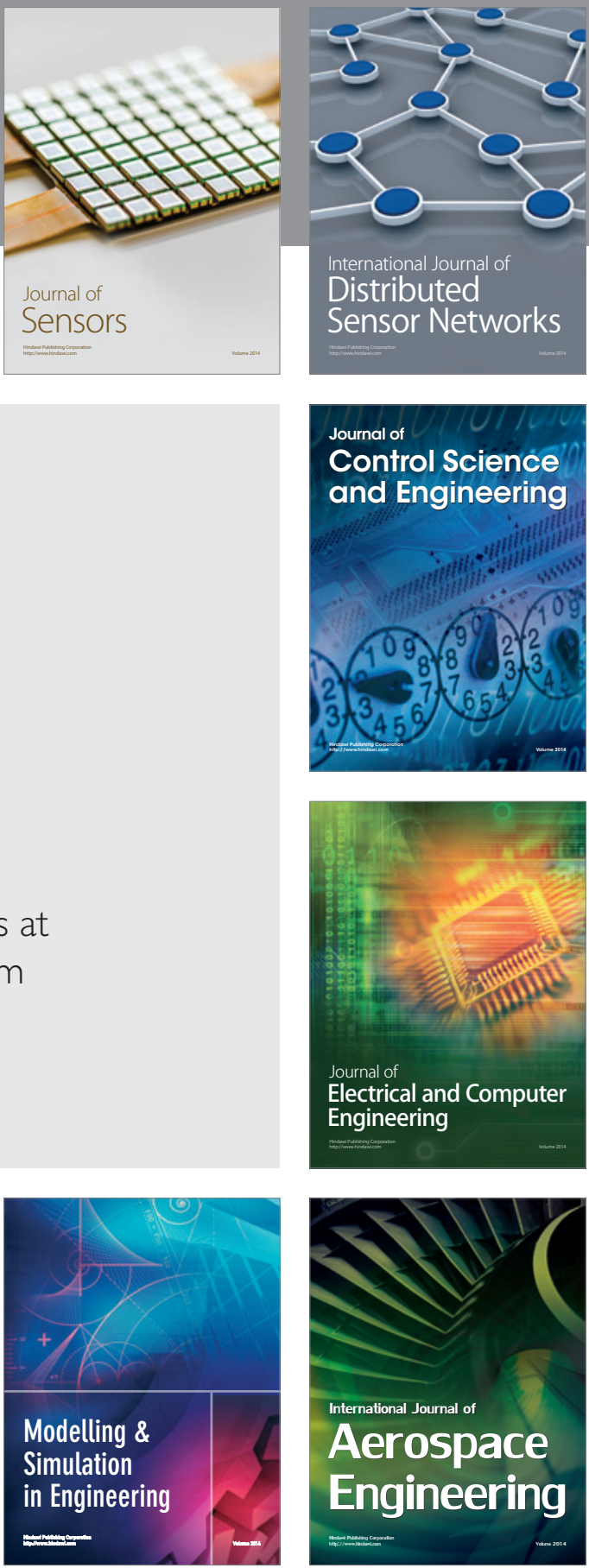

Journal of

Control Science

and Engineering
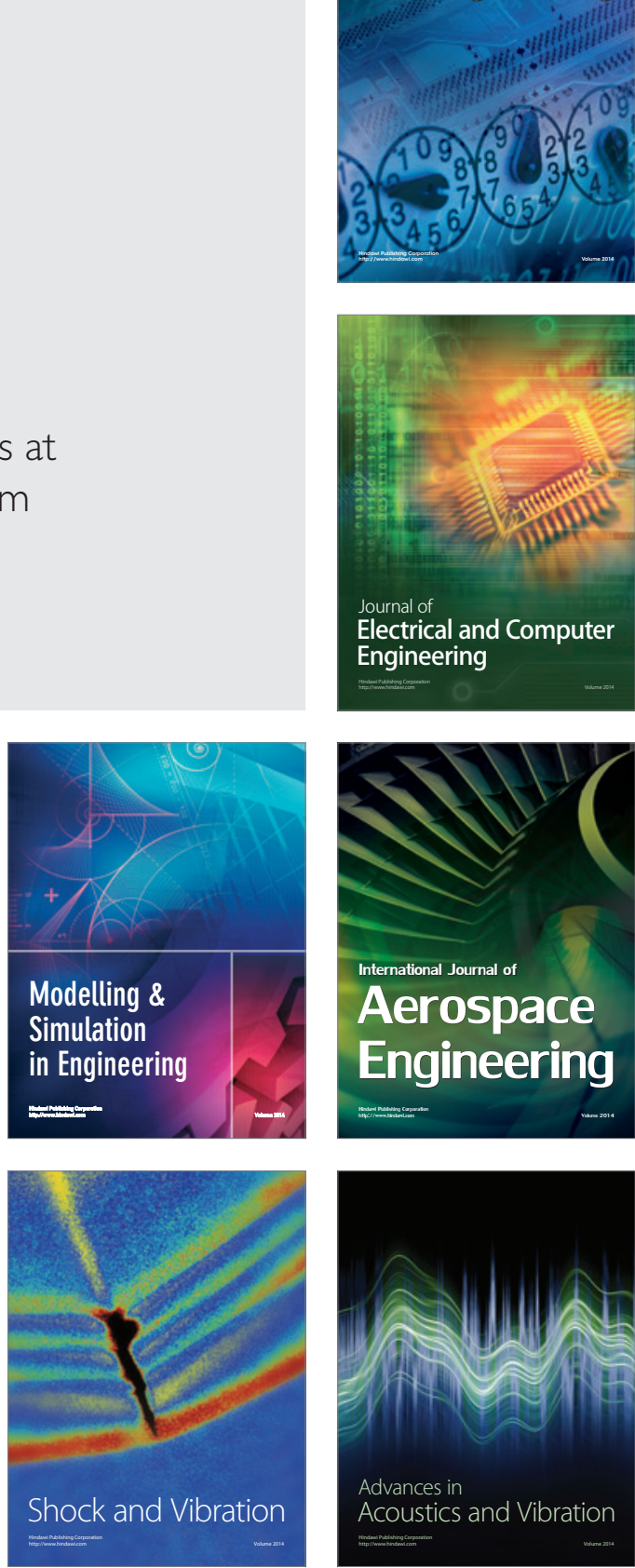